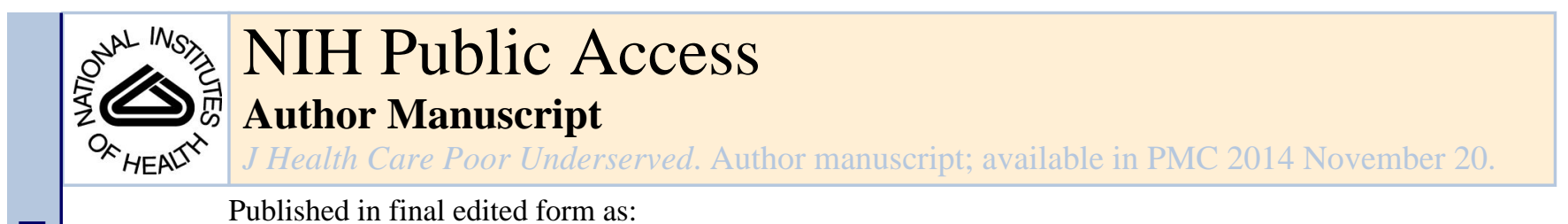

Published in final edited form as:

J Health Care Poor Underserved. 2012 August ; 23(3): 1205-1221. doi:10.1353/hpu.2012.0090.

\title{
Oral Health Activities of Early Head Start and Migrant and Seasonal Head Start Programs
}

\author{
Ashley M. Kranz, BA ${ }^{1}$, R. Gary Rozier, DDS, MPH ${ }^{1}$, Leslie P. Zeldin, MPH, MSUP ${ }^{1}$, and John \\ S. Preisser, $\mathrm{PhD}^{2}$ \\ ${ }^{1}$ Department of Health Policy and Management, Gillings School of Global Public Health, \\ University of North Carolina at Chapel Hill \\ ${ }^{2}$ Department of Biostatistics, Gillings School of Global Public Health, University of North Carolina \\ at Chapel Hill
}

\section{Abstract}

Guidelines recommend that Migrant and Seasonal Head Start programs (MSHS) address the dental needs of children of migrant and seasonal farmworkers. This study describes parent- and child-oriented oral health activities of North Carolina's MSHS programs and compares them with non-migrant Early Head Start (EHS) programs using data collected from a questionnaire completed by teachers and family services staff. MSHS staff reported engaging in more oral health activities than EHS staff, which was confirmed by results of logit and ordered logit regression models. Despite promising findings about the engagement of MSHS staff, participation in oral health activities is lower than recommended. Differences between EHS and MSHS programs might be due to differing needs of enrolled children and families or to different approaches to meeting the needs of families.

\section{Keywords}

oral health; migrant farmworkers; head start; early intervention

\begin{abstract}
Poor oral health among young children in the United States (U.S.) is a major concern. Early childhood caries (ECC), defined as one or more decayed, missing or filled teeth for children younger than seven years of age, is the most common chronic disease of childhood, affecting more than four million children nationwide. ${ }^{1,2}$ Among children two to four years old, the prevalence of dental caries has increased and surveys show that fewer than half of young children had a dental visit in the previous year. ${ }^{2}$ Young children living in poverty are
\end{abstract}

Copyright (C) 2012 Meharry Medical College.

Corresponding Author: Ashley Kranz, Department of Health Policy and Management, UNC Gillings School of Global Public Health, CB \#7411, Chapel Hill, NC 27599, Phone: 248-229-0701, Fax: 919-966-6961, akranz@ unc.edu, http://www.sph.unc.edu/hpaa/. Reprints: Send request for reprints to corresponding author.

Disclaimers: None

Preliminary Presentation: Poster presented under the title "Oral Health Activities of Migrant and Seasonal Head Start Programs" at the International Association for Dental Research Annual Meeting, March 16, 2011, San Diego, CA

The content is solely the responsibility of the authors and does not necessarily represent the official views of the NIDCR or the

National Institutes of Health. 
twice as likely to experience caries yet only half as likely to visit a dentist compared to higher income children. ${ }^{3}$

Like other low-income populations, the children of migrant and seasonal farmworkers (MSFW) experience dental disease at a high rate and face challenges to obtaining dental care. Prior studies have found that school-age children of MSFWs are more likely to experience dental caries and to have untreated oral health problems than other children. ${ }^{4-6} \mathrm{~A}$ study conducted in North Carolina (NC) found that 39.3\% of children of MSFWs had never visited the dentist or only did so because of an emergency. ${ }^{7}$ Access to dental care is further impeded by the rural residency of MSFWs. In rural areas, children in low-income families utilize dental services less and report more unmet dental needs than their counterparts in non-rural areas. ${ }^{8}$

Early childhood is an important time for oral health promotion. Based on recommendations from the American Academy of Pediatric Dentistry (AAPD), parents should begin performing good oral hygiene practices for their child soon after birth, feed them a noncariogenic diet and have the first professional oral health risk assessment by 12 months of age. ${ }^{9}$ For young children living in poverty, Early Head Start (EHS) programs help families achieve these oral health benchmarks. EHS addresses the social, educational and health needs of children three years of age and younger. Programs adhere to federal performance standards issued by the Office of Head Start, which outline teacher qualifications, safety procedures, and health promotion activities, among other things. Guidelines emphasize both the prevention and treatment of oral health problems in young children. ${ }^{10}$ Programs are directed to clean children's mouths once daily after meals following age-specific recommendations and help families identify a regular dental provider for their children. In 2011, EHS programs will serve nearly 100,000 children and families nationwide. ${ }^{11}$

Young children of MSFWs are eligible for early education and childcare services through the Migrant and Seasonal Head Start (MSHS) program. MSHS follows the same performance standards as EHS, but tailors its services to the specific cultural and lifestyle needs of MSFWs and their families. Because MSFWs move frequently to obtain seasonal work, MSHS programs typically operate only during agricultural harvest seasons, from two to ten months annually depending on the crop and the area.. ${ }^{12-14}$ To prevent families from bringing children into the fields where they would risk exposure to environmental dangers, MSHS programs provide seasonal child care from early in the morning until late in the evening at no cost to MSFWs. ${ }^{15}$ MSHS programs annually serve 35,657 children ages zeroto-five years old at more than 550 sites in 38 states. ${ }^{12}$

The East Coast Migrant Head Start Program (ECMHSP) provides direct services and contracts with delegate agencies in ten states in the eastern migrant stream. ${ }^{16}$ MSFWs in this stream generally begin the season in Florida and move north, eventually ending in New York or Maine. ${ }^{17}$ About $75 \%$ of MSFWs in the eastern stream are from Mexico and of these Mexican-born MSFWs, only 5\% speak and read English. ${ }^{13}$ In FY2010-11, ECMHSP anticipates serving 4,964 children with more than $\$ 53$ million in funding from the Office of Head Start. ${ }^{16}$ 
ECMHSP and EHS have identified oral health as a priority for children and their families. In 2003, 26\% of ECMHSP children examined by a dentist were in need of treatment and an ECMHSP community assessment found dental care to be the most commonly reported health need of families (ECMHSP, Oral health initiative grant). In 2007 the Office of Head Start revised their oral health program instructions to emphasize the role that all Head Start programs can play in improving the oral health of young children. ${ }^{10}$ The following year the Office of Head Start partnered with the AAPD in a national initiative to help young children establish a dental home. ${ }^{18}$

Despite prioritizing oral health, limited research exists regarding the oral health activities of EHS and MSHS. Two studies have examined the dental content of Head Start guidelines, which EHS and MSHS follow. ${ }^{19,} 20$ Two more studies examining dental health practices included, but did not solely focus on children enrolled in MSHS programs. ${ }^{21,22}$ The first study, conducted in migrant dental clinics in Chicago, examined the dental health practices of MSFWs and their preschool children finding that among the children enrolled in Head Start, $79 \%$ had visited a dentist in the past year, compared to only $36 \%$ of children not enrolled in Head Start. ${ }^{21}$ An ethnographic study conducted in central Florida by Castañeda, Carrion, Kline, et al. found that nearly all MSFW parents interviewed, including parents with children enrolled in MSHS, reported brushing their children's teeth daily and almost one-third were knowledgeable about the recommended age one dental visit. ${ }^{22}$ Based on findings from interviews, the authors suggest that social class and structural barriers such as Medicaid reimbursement policies and dental professional shortages, rather than knowledge, culture, or ethnicity, contribute to the unmet dental needs of MSFWs.

Few studies have examined the oral health activities occurring within EHS and to our knowledge none have studied these specific activities in MSHS. We aim to fill this information gap about the oral health activities of MSHS by comparing the activities of EHS and MSHS using EHS as a point of reference because both programs follow the same performance standards and serve children at elevated risk of having poor oral health. This comparison is important, because not only does program performance need to be determined, but the extent to which children of MSFW receive dental services, a subgroup of low-income children at particularly elevated risk for dental disease, needs to be known to help plan interventions.

To better understand the oral health activities occurring within EHS in anticipation of a planned oral health intervention, a survey of EHS staff in NC was conducted in 2005. The survey was extended to MSHS programs in NC after MSHS staff expressed interest in participating. NC provides a good environment for examining MSFW families because from 1990-2000 the number of Latino immigrant children under five years of age increased by 400\%. ${ }^{17}$ Additionally, like the rest of the country, $\mathrm{NC}$ faces challenges with the prevalence of dental disease and dental professional shortages. ${ }^{23,} 24 \mathrm{~A}$ recently published analysis of this EHS survey of non-migrant programs found that the level of oral health activity in EHS programs did not fully meet recommended federal performance standards at the time of the survey. ${ }^{25}$ Our current study expands on prior research by examining MSHS staff members' oral health activities directed toward parents and children ages three years old and younger and compares those activities to non-migrant EHS staff members. The objectives of our 
study are to describe the likelihood of MSHS staff engaging in oral health activities and to compare their reported level of activity with non-migrant EHS programs in the state.

\section{Methods}

\section{Data source}

A cross-sectional census was conducted in NC EHS programs in June 2005 and in MSHS programs in September 2005 with staff who work with children three years old and younger. All EHS programs $(n=18)$ and all MSHS programs $(n=10)$ in NC participated in the survey. For EHS programs, research staff delivered questionnaires in person to a designated EHS staff member in each program who distributed questionnaires. EHS staff members completed questionnaires, which were later collected and returned via FedEx by the designated staff member. For MSHS programs, questionnaires were distributed at a meeting of all program directors, who were instructed to share with staff working with children three years old and younger. Completed surveys were subsequently collected and mailed to the study investigators by a designated staff member. The study was approved by the Institutional Review Board at the University of North Carolina at Chapel Hill and the NC Head Start State Collaboration Office.

As previously described by Mathu-Muju, Lee, Zeldin et al., questionnaires were developed using guidance from several sources, including: the overall study objectives, focus groups with parents and staff, Head Start program performance standards, and previously developed and tested questionnaires. ${ }^{25}$ Questionnaires included six domains related to classroom oral health activities (knowledge, value placed on oral health, confidence in performing dental activities, expected outcomes, current practices, and barriers encountered); items about interactions with families; and items for dental screening, referral, and follow-up of children. Additional details about the sample selection, questionnaire development and data collection are described in previous papers. ${ }^{25,26}$ For this study, variables were created from the aforementioned survey and regression models were used to examine the likelihood of MSHS staff engaging in oral health activities, to compare their reported level of activity with EHS staff, and to examine associations between independent variables and level of oral health activity.

\section{Dependent variables}

Eight binary variables indicating participation in oral health activities were derived from questions asking about the frequency (5-point scale of never to very frequently) with which each activity was conducted. Reponses of "frequently" or "very frequently" were recoded to indicate participation in the oral health activity. Among these questions four items described oral health activities related to tooth brushing and classroom education and four items described parent-focused activities. Items related to brushing asked about who was brushing the child's teeth in the classroom, if toothpaste was used, and if classroom dental health education was provided to children. Items related to parent-focused oral health activities asked about conversations regarding oral health practices at home and the dental health of children and parents. These items were later summed to create two variables providing 
counts of brushing-related and parent-focused oral health activities (ranges $=0-4$ for each variable).

\section{Independent variables}

Because the objective of this study is to determine the level of oral health activity in MSHS and use EHS as a point of reference, the main explanatory variable is a binary variable indicating whether the teacher worked in a MSHS program or not. Additional independent variables were used to control for other factors that might affect participation in oral health activities.

A binary variable was constructed to indicate whether a teacher received training on how to include dental health in their program activities. Two binary variables measuring oral health knowledge were constructed using answers to questions that asked if toothpaste should cover all the bristles of a child's toothbrush (disagree) and if low-income children were less likely to develop caries (disagree).

Two multi-item scales were constructed to measure teachers' perceived oral health selfefficacy and value placed on oral health. Perceived oral health self-efficacy was assessed using 12 survey questions related to staff members' confidence in their ability to perform oral health activities and the expected outcomes of those activities. Questions used 0-4 Likert-type scale responses, which were summed to create a multi-item scale with higher values indicating greater perceived oral health self-efficacy (range $=0-48$; mean $=30.1$; Cronbach's alpha $=0.80$ ). The value teachers placed on oral health was measured using 15 survey questions, which asked about the importance of primary teeth, dental visits, and oral health activities at the program level. Most questions used 0-4 Likert-type response scales, which were recoded to binary items (agree vs. disagree) and summed to create a multi-item scale (range $=0-15$; mean $=11.4$; Cronbach's alpha $=0.80$ ). Ordinal variables (low, medium, high) were constructed based on the $25^{\text {th }}$ and $75^{\text {th }}$ percentiles of the self-efficacy and value multi-item scales.

To assess barriers to providing dental activities for children and parents, staff were provided a list of 12 potential individual- and community-level barriers with 0-4 Likert-type scales and asked to indicate how much each one was an obstacle. Responses of "very much an obstacle" and "somewhat an obstacle" were recoded to indicate a staff reported barrier to dental activities, and then summed to create a count of the total barriers (range=0-12).

Control variables included race/ethnicity, educational attainment level, and the number of years employed by Head Start.

\section{Data completeness}

The primary analysis was limited to staff self-identifying as teachers or family services staff (i.e., home visitors and family services coordinators), which led to the exclusion of 103 EHS and 29 MSHS staff questionnaires. We limited analysis of brushing activities to teachers because they regularly interact with children in the classroom. We included family services staff members such as home visitors in addition to teachers in the analysis of parent-focused activities because of their importance in providing oral health services for adults in the 
family. If a staff member had a missing response for a single item that comprised a multiitem construct (count of dependent variable, value placed on oral health, oral health selfefficacy, or barriers to oral health activities), the response for that question was imputed as the average score across non-missing items from that construct and rounded to the nearest whole number.

\section{Analytical approach}

Following construction of variables, multicollinearity was explored by analyzing pairwise correlation. Descriptive statistics were calculated for all variables. Preliminary bivariate analyses examining differences between staff in MSHS and EHS programs were conducted using t-tests for continuous variables and chi-square tests for categorical variables, without adjustment for a potential clustering effect of staff within programs.

Logistic regression models for dichotomous outcomes were used to estimate the odds of participating in each oral health activity. Z-tests were used to examine the association between staff in MSHS compared to EHS regarding the odds of engaging in each individual oral health activity, holding all other variables constant. Ordered logit regression models were used to model the probabilities for the number $(0-4)$ of brushing and parent-focused oral health activities for each staff member. ${ }^{27}$ To assess the proportional odds assumption, likelihood ratio tests were calculated. ${ }^{28} \mathrm{Z}$-tests were used to examine the associations between independent variables and the number of oral health activities in which staff engage. Results of the ordered logit regression models are interpreted as the odds of engaging in more oral health activities versus fewer activities, holding all other variables constant.

As recommended by Cameron and Trivedi, clustered standard errors were used in all models to adjust for intra group correlation due to staff clustering within programs. ${ }^{29}$ Analyses were performed using Stata 10 (StataCorp, College Station, TX), and tests were conducted using a significance level of 0.05 .

\section{Results}

Staff included in the analysis of parent-focused oral health activities ( $N=401$ : $E H S=329$, MSHS=72) were a subset of the 450 eligible staff respondents (response rates: EHS=98.8\%, MSHS $=97.6 \%$ ). After imputation of missing items for approximately $10 \%$ of staff members, 49 respondents were omitted from the analysis because the number of missing items did not meet the item response criteria for imputation of missing values. Because brushing activities are expected to occur within the classroom and recommendations for cleaning the mouths of infants differ from recommendations for older children, analysis of brushing related activities was limited to staff who self-reported as teachers who work with children 12 months and older $(\mathrm{N}=300, \mathrm{EHS}=250, \mathrm{MSHS}=50) .{ }^{9}$ No statistically significant differences were observed for oral health activities between staff with missing and non-missing variables. 


\section{Sample characteristics}

As displayed in Table 1, an average staff member in this sample was white, had some college education, and was employed by EHS or MSHS for three or more years. Staff placed a moderate value on oral health, had a medium level of perceived oral health self-efficacy, and were knowledgeable about low-income children's increased risk of developing caries and the amount of toothpaste to use when brushing.

Using bivariate analysis techniques we observed significant differences between staff in EHS and MSHS programs for several characteristics. Unlike staff in EHS (37.1\%), a majority of MSHS teachers (55.3\%) had received dental training from Head Start.

Significant differences were also observed regarding knowledge of the amount of toothpaste to use when brushing, the number of years employed by Head Start, educational attainment level, and the racial/ethnic background of staff.

\section{Individual oral health activities}

The percentages of EHS and MSHS staff performing each of the individual oral health activities frequently and very frequently are presented in Table 2 along with the estimated odds ratio comparing the performance of MSHS teachers to that of EHS teachers for each activity. Descriptive analyses suggested that among parent-focused oral health activities, staff were most likely to report advising parents on cleaning their child's teeth (EHS=38\%, MSHS $=66.7 \%$ ) and talking to parents about food choices to promote oral health (EHS $=39.5 \%$, MSHS $=62.5 \%)$. Among activities related to brushing, most teachers (EHS $=84.4 \%$, MSHS $=96 \%)$ had children brush their own teeth and most MSHS teachers provided classroom education about dental health (EHS $=45.2 \%$, MSHS $=74 \%)$. The results of chi-square tests suggested that MSHS staff were significantly more likely than EHS staff to engage in all parent-focused activities and nearly all brushing activities.

\section{Binary outcome models}

Among parent-focused oral health activities, MSHS staff compared to EHS staff had statistically significant greater odds of engaging in oral health activities, holding all other variables constant (Table 2). Regarding brushing-related activities, MSHS teachers compared to EHS teachers had greater odds of having children brush their own teeth, using toothpaste, and providing classroom education about dental health.

\section{Count of oral health activities}

On average, staff in MSHS programs participated in 2.18 ( $\mathrm{SD}=1.60)$ parent-focused oral health activities and $2.86(\mathrm{SD}=0.90)$ brushing activities compared to $1.28(\mathrm{SD}=1.45)$ and 2.02 ( $\mathrm{SD}=1.10$ ), respectively, for EHS staff members (Table 1). Table 3 provides the observed probability distribution of the count of an individual's oral health activities. Among MSHS staff, approximately $49 \%$ reported participating in three or more parentfocused activities and $68 \%$ reported participating in three or more brushing-related activities. Among EHS staff, 24\% reported participating in three or more parent-focused activities and $34 \%$ reported participating in three or more brushing-related activities. 


\section{Ordered outcome models}

Initially, the distributions of observed proportions of the count of oral health activities in Table 3 are inspected to determine if the association of the MSHS-EHS variable and the number of oral health activities depends on how a cutpoint is applied to the latter in making it a dichotomous variable. ${ }^{27}$ Beginning with the dichotomy comparing zero oral health activities to one or more activities, and then progressing through all other possible dichotomizations (e.g., 0 or 1 versus 2 or more activities, etc), the observed odds ratios for parent-focused oral health activities for all possible cutpoints are 2.48, 2.83, 3.04, and 3.20, respectively. Similarly, noting that a zero observed frequency precludes the calculation of an observed odds ratio for the first dichotomy for brushing related activities, the remaining odds ratios are 5.41, 3.98 and 3.83. The similarity of these values is consistent with the proportional odds assumption for the comparison of MSHS versus EHS. Formally, for both dependent variables, likelihood ratio tests failed to reject the proportional odds assumption for all independent variables assessed simultaneously (Parent activities: $\mathrm{P}=0.18$; Brushing: $\mathrm{P}=0.16)$. Thus proportional odds ordered logit models were used to estimate the effects of the independent variables on both outcomes.

Parent-focused activities-As illustrated by Table 3, an average MSHS staff member had a 0.84 predicted probability of engaging in two or more parent-focused oral health activities compared to 0.33 for EHS staff. Odds ratios for the association of independent variables with teachers' oral health activities are provided in Table 4. MSHS staff had 2.03 times greater odds of engaging in more parent-focused oral health activities than EHS staff, holding all other variables constant $(95 \% \mathrm{CI}=1.26,3.26)$. Receipt of dental training was significantly associated with 2.11 times greater odds of engaging in more activities (95\% $\mathrm{CI}=1.31,3.38$ ). Placing moderate or high value on oral health or having high self-efficacy compared to low was associated with greater odds of engaging in more parent-focused oral health activities.

Brushing-related activities ordered outcome model-As illustrated by Table 3 , an average MSHS teacher had a higher predicted probability of participating in all four brushing related activities than an EHS teacher (MSHS=0.50, EHS=0.07). MSHS teachers had a 0.97 predicted probability of engaging in two or more activities related to brushing, whereas the predicted probability for EHS teachers was 0.70 .

As displayed in table 4, MSHS teachers had 3.65 times greater odds of engaging in more brushing related activities than EHS teachers, holding all other variables constant (95\% $\mathrm{CI}=1.69,7.89$ ). Teachers who had received dental training had 2.33 times greater odds of engaging in more brushing related activities $(95 \% \mathrm{CI}=1.50,3.62)$. Having high perceived oral health self-efficacy compared to low was associated with 2.11 times greater odds of engaging in more brushing related activities $(95 \% \mathrm{CI}=1.23,3.90)$.

\section{Discussion}

EHS and MSHS help promote the oral health of low-income children and families through classroom tooth brushing and discussions with families about good oral health practices. Staff members' participation in these activities is important to examine because low-income 
children disproportionally suffer from ECC and encounter barriers to dental services, thus targeting this group may help to lessen oral health disparities. Our findings suggest that EHS and MSHS staff members are very similar in many oral health-related beliefs. They reported comparable levels of value placed on oral health, perceived oral health self-efficacy, and barriers to oral health activities. However, participation in oral health activities differed between MSHS and EHS programs, with MSHS staff being more likely to participate in all oral health activities. Less than half of all non-migrant EHS staff reported participating in any single oral health activity, excluding having children brush their own teeth. Moreover, working in a MSHS program was associated with greater odds of participating in nearly all brushing related activities.

Based on findings from this analysis and existing literature, we can hypothesize why participation rates in oral health-related activities differ between EHS and MSHS. First, the variation observed between EHS and MSHS programs can be indicative of different priorities and needs among the enrolled children and families. Programs conduct community assessments every three years to identify the specific needs of the area they serve. Thus, EHS and MSHS may prioritize different activities in an attempt to meet the distinct needs of their communities. For example, an East Coast Migrant Head Start Program (ECMHSP) community assessment conducted in 2003 indicated dental care was the most commonly reported health need of families (ECMHSP, Oral health initiative grant). Descriptive analysis in our study indicated that MSHS staff were significantly more likely to have received dental health training.

Furthermore, participation in oral health activities may be different because of the approaches used to meeting the needs of families. MSHS must accommodate the work schedules of MSFW parents, generally staying open for extended daytime hours, but only for a few months a year, whereas EHS programs are generally open year round for five to six hours daily. These different schedules may affect the oral health activities of young children in several ways. MSHS programs must act immediately to get all needs met before families move to another location. Children enrolled in EHS are with the program for the entire year, thus EHS can approach the health and education needs of children more deliberately within guidance provided by the federal standards. For example, staff have 90 days to get the oral health assessments completed..$^{10}$ Because families are transient, MSHS programs have the additional challenge of establishing dental homes and maintaining continuity of care, practices recommended by the AAPD and the Office of Head Start. ${ }^{18}$ Extended daytime hours provide MSHS teachers with more opportunities to engage in oral health activities in the classroom than EHS, which may help to explain the difference in brushing-related activities reported by the two program types. Tooth brushing with young children can be time consuming because of the frequency and supervision recommendations in hygiene guidelines. ${ }^{30-32}$

Despite promising findings in this study about the engagement of MSHS programs in oral health activities, participation is still lower than desired. Out of the four parent-focused oral health activities we examined, MSHS staff participated in 2.18 activities on average. And while family services staff reported participating in these activities more often than teachers (2.63 activities), it was still at a level below what is recommended in the performance 
standards. Oral health activities directed towards parents of children enrolled in MSHS are important because these children are generally served by a program for a few months a year, meaning staff have a limited amount of time to educate parents about the importance of their child's oral health. The study by Castañeda and colleagues found that MSFW parents were knowledgeable about child oral health, but that they encountered challenges in putting their knowledge into practice. ${ }^{22}$ In 2006, the Office of Head Start provided funds to $52 \mathrm{Head}$ Start, EHS, and MSHS programs for oral health promotion activities. ECMSHSP received one of these grants and used it to develop Spanish language training materials to facilitate parental education about oral health promotion and to implement a training program to educate staff. A final report on this grant initiative concluded that educating staff, children, and parents about the importance of oral health is a promising strategy with the potential to positively affect children's oral health and can be successfully implemented across programs. ${ }^{33}$ Specifically, the report recommended that oral health education be sensitive to parents' language, culture, and reading level and be incorporated into meetings and workshops. Furthermore, the Association of State and Territorial Dental Directors (ASTDD) encourages partnerships between Head Start and state oral health programs to increase oral health knowledge and to facilitate access to oral health services. ${ }^{34}$

Few staff members reported asking parents if their child's dental health needs were being met. Whereas nearly all children in EHS programs are eligible to have medical and dental services reimbursed by Medicaid, MSHS programs may encounter barriers to care due to lack of Medicaid portability. ${ }^{35}$ Medicaid portability refers to the ability to use the benefits of one state's Medicaid program in another state, which is essential for the children of MSFW who frequently move. Different enrollment processes and forms, in addition to lengthy determinations of eligibility, may deter or inhibit MSFWs from obtaining public health insurance as they move from state-to-state. ${ }^{36}$ Conversations with parents about their own oral health were also infrequent in both program types. This finding may also be related to dental insurance status because adult benefits in Medicaid are limited in many states. Lack of health insurance is an acute concern among MSHS parents. A study conducted in 2000 reported that $85 \%$ of MSFWs lacked health insurance. ${ }^{37}$ Furthermore, a study of MSFW families in NC found that $73 \%$ of children had visited the dentist in the last year compared to only $47 \%$ of their mothers. ${ }^{7}$

Most teachers reported having children brush their own teeth in the classroom (EHS=84\%, MSHS=96\%), but a much smaller proportion indicated they brush children's teeth for them (EHS $=30 \%$, MSHS $=40 \%$ ). Brushing guidelines for EHS programs developed by the National Maternal \& Child Oral Health Resource Center recommend that after the child brushes his or her teeth in the classroom, the teacher should brush the child's teeth as well to ensure proper cleaning. ${ }^{31}$ Both the child and teacher should be engaged in tooth brushing because young children do not have the motor skills to effectively clean their own teeth. Fewer than half of EHS teachers (41.4\%) reported using toothpaste. Whereas 76\% of MSHS teachers reported using toothpaste, only $48.6 \%$ of MSHS teachers were knowledgeable about the amount of toothpaste to use when brushing children's teeth. The effectiveness of use of fluoridated toothpaste in preventing dental caries in young children is welldocumented and a practice supported by the Office of Head Start. ${ }^{10,38}$ Supervised brushing 
and use of fluoridated toothpaste are important practices that can help achieve desired oral health outcomes.

\section{Limitations}

Any changes these programs since 2005 are not captured in this analysis and results might not reflect current practices. We included all MSHS programs in NC, but we may not have surveyed all staff because questionnaires were distributed in September after some MSFW families had already left for the season and programs were adjusting to their winter schedules. During follow-up telephone interviews conducted with program directors, one MSHS program indicated that all staff $(n=8)$ work with children of all ages, meaning that the responses of staff in one program may have described the oral health activities of older children. Furthermore, because the data were collected from self-completed questionnaires, bias may be introduced if individuals incorrectly stated their level of participation in oral health activities. Finally, findings should not be interpreted causally because of the crosssectional design of this study.

\section{Summary}

Although EHS and MSHS serve different populations, both programs are committed to promoting the oral health of young children from low-income families. Based on our findings, we conclude that both MSHS and EHS staff are more likely to engage in brushing activities in the classroom than in parent-directed oral health activities. Overall, MSHS staff reported engaging in more oral health activities than EHS staff, and they were more likely to participate in most brushing-related activities. Because of the higher level of engagement of MSHS in oral health activities compared to EHS, further research into the strategies employed by MSHS might prove useful in designing interventions. Furthermore, research examining the effect of oral health initiatives, such as the ones funded by grants from the Office of Head Start, may provide evidence of effectiveness for intervention strategies.

\section{Acknowledgments}

Support: Supported by Grant No. 11-P-91251/4-02 from CMS, HRSA, and CDC and Grant No. R01 DE018236 from the National Institute of Dental and Craniofacial Research.

This project was supported by Grant No. 11-P-91251/4-02 from CMS, HRSA, and CDC and Grant No. R01 DE018236 from the National Institute of Dental and Craniofacial Research (NIDCR).

\section{References}

1. Council on Clinical Affairs, American Academy of Pediatric Dentistry. Definition of early childhood caries. Chicago (IL): American Academy of Pediatric Dentistry; 2009. Available at http:// www.aapd.org/media/policies.asp

2. Dye BA, Tan S, Smith V, et al. Trends in oral health status. United States, 1988-1994 and 19992004. Vital Health Stat. 2007 Apr; 248(11):1-92.

3. Edelstein B. Disparities in oral health and access to care: findings of national surveys. Ambul Pediatr. 2002; 2(2):141-147. [PubMed: 11950385]

4. Koday M, Rosenstein DI, Lopez GM. Dental decay rates among children of migrant workers in Yakima, WA. Public Health Rep. 1990; 105(5):530-3. [PubMed: 2120733]

5. Call RL, Entwistle B, Swanson T. Dental caries in permanent teeth in children of migrant farm workers. Am J Public Health. 1987; 77(8):1002-3. [PubMed: 3605464] 
6. Woolfolk M, Hamard M, Bagramian RA, et al. Oral health of children of migrant farm workers in northwest Michigan. J Public Health Dent. 1984; 44(3):101-105. [PubMed: 6592350]

7. Quandt SA, Clark HM, Rao P, et al. Oral health of children and adults in Latino migrant and seasonal farmworker families. J Immigr Minor Health. 2007; 9(3):229-235. [PubMed: 17252193]

8. Hartsock LG, Hall MB, Connor AM. Informing the policy agenda: The community voices experience on dental health for children in North Carolina's rural communities. J Health Care Poor Underserved. 2006; 17(1S):111-123. [PubMed: 16520519]

9. Infant Oral Health Subcommittee, Clinical Affairs Committee. Guideline on infant oral health care. Chicago, IL: American Academy of Pediatric Dentistry; 2009. Available at http://www.aapd.org/ media/Policies_Guidelines/G_InfantOralHealthCare.pdf

10. Office of Head Start. DHHS Log no. ACF-PI-HS-06-03. Washington, DC: Administration for Children and Families; 2006. Oral health revision. Available at http://eclkc.ohs.acf.hhs.gov/hslc/ Head\%20Start\%20Program/Program\%20Design\%20and\%20Management/Head\%20Start \%20Requirements/PIs/2006/resour_pri_00109_122006.html

11. Office of Head Start. Head start program fact sheet: fiscal year 2010. Washington, DC: Administration for Children and Families; 2010. Available at http://www.acf.hhs.gov/ programs/ohs/about/fy2010.html

12. Cuesta, G. National migrant \& seasonal head start collaboration office annual report. Washington, DC: Academy for Educational Development; 2008. Available at http://ece.aed.org/publications/ nms-NewslettersandReports.html

13. Emmi KE, Jurkowski JM, Codru N, et al. Assessing the health of migrant and seasonal farmworkers in New York state: statewide data 2003-2005. J Health Care Poor Underserved. 2010; 21(2):448-463. [PubMed: 20453349]

14. Boss, J. Migrant head start services for infants and toddlers. Washington, DC: Head Start Bulletin, Office of Head Start; 2000. Available at http://eclkc.ohs.acf.hhs.gov/hslc/ecdh/eecd/FamiliesParent\%20Involvement/Support\%20for\%20Home\%20Culture\%20and\%20Diversity/ edudev_art_00213_072505.html

15. Hansen E, Donohoe M. Health issues of migrant and seasonal farmworkers. J Health Care Poor Underserved. 2003; 14(2):153-164. [PubMed: 12739296]

16. 2009 Annual Report. Arlington, VA: East Coast Migrant Head Start Project; 2009. Available at http://www.ecmhsp.org/default/2009\%20Annual\%20Report.pdf

17. National Migrant and Seasonal Head Start Collaboration Office. Invisible children. Washington, DC: Academy for Educational Development; 2009. Available at http://ece.aed.org/ publications/nms/InvisibleChildren_2.18.09.pdf

18. Crall, J.; Silverman, J. AAPD-OHS dental home initiative overview: partnering to provide dental homes and optimal oral health for HS/EHS children throughout the U.S. Presented at: National Oral Health Conference; Portland (OR). April 2009;

19. Kranz AM, Rozier RG. Oral health content of early education and child care regulations and standards. J Public Health Dent. 2011; 71(2):81-90. [PubMed: 21070244]

20. Edelstein B. Access to dental care for Head Start enrollees. J Public Health Dent. 2000; 60(3):221229. [PubMed: 11109221]

21. Lukes SM. Oral health knowledge attitudes and behaviors of migrant preschooler parents. J Dent Hyg. 2010; 84(2):87-93. [PubMed: 20359420]

22. Castaneda H, Carrion IV, Kline N, et al. False hope: effects of social class and health policy on oral health inequalities for migrant farmworker families. Soc Sci Med. 2010; 71(11):2028-37. [PubMed: 20943298]

23. Rozier R, King R. Defining the need for dental care in North Carolina: contributions of public health surveillance of dental diseases and conditions. NC Med J. 2005; 66(6):438-44.

24. Stamm JW. The dentist workforce in North Carolina 2005: a commentary. NC Med J. 2005; 66(6): 445-51.

25. Mathu-Muju KR, Lee JY, Zeldin LP, et al. Opinions of early head start staff about the provision of preventive dental services by primary medical care providers. J Public Health Dent. 2008; 68(3): 154-62. [PubMed: 18843804] 
26. Kranz AM, Rozier RG, Zeldin LP, et al. Oral health activities of early head start teachers directed toward children and parents. J Public Health Dent. 2011; 71(2):161-169. [PubMed: 21774140]

27. Preisser JS, Phillips C, Perin J, et al. Regression models for patient-reported measures having ordered categories recorded on multiple occasions. Community Dent Oral Epidemiol. 2011; 39(2): 154-163. [PubMed: 21070317]

28. Long, SL.; Freese, J. Regression models for categorical dependent variables using Stata. 2. College Station, TX: StataCorp LP; 2006. Chapter 5: models for ordinal outcomes; p. 183-213.

29. Cameron, AC.; Trivedi, PK. Microeconomics Using Stata. College Station, TX: StataCorp LP; 2009. Chapter 15: Multinomial models; p. 445-448.

30. American Academy of Pediatrics, American Public Health Association, National Resource Center for Health Safety in Child Care. Caring for our children: National health and safety performance standards: Guidelines for out-of-home child care. Elk Grove Village (IL): American Academy of Pediatrics; 2002. Chapter 3: health promotion and protection in child care.

31. Bickel HW Jr. Toothbrushing and head start: What's it all about? Head Start Bulletin. 2001; 71:27-28. Available at http://eclkc.ohs.acf.hhs.gov/hslc/resources/ECLKC_Bookstore/PDFs/ 421CEF928C56391D1041DFCE1F0119AC.pdf.

32. Office of Oral Health. Growing healthy smiles in the child care setting. Boston, MA: Massachusetts Department of Public Health; 2009. Available at: http://www.mass.gov/Eeohhs2/ docs/dph/com_health/oral_grow_healthy_smiles_child_care.pdf

33. Del Grosso, P.; Brown, A.; Silva, S., et al. Mathematica Policy Research, Inc., Princeton, NJ. HHS Contract no. 233-02-0086. Washington (DC): Administration for Children and Families; 2008. Strategies for promoting prevention and improving oral health care delivery in head start: findings from the oral health initiative evaluation.

34. Geurink, K.; Isman, B. Head start oral health project: evaluation report 2001-2008. Sparks, NV: Association of State and Territorial Dental Directors; 2009.

35. Rosenbaum, S. Medicaid portability in the context of oral health care for Head Start-enrolled children in migrant farmworker families. Prepared for an Oral Health Expert Meeting sponsored by the Maternal and Child Health Bureau, in partnership with the Migrant and Seasonal Head Start Collaboration Office; Rockville (MD). March 2008; Available at: http://www.mpca.net/Client/ MPCA/Files/MedicaidPortability_Report04.pdf

36. Centers for Medicare \& Medicaid Services. Report to Congress: study regarding barriers to participation of farmworkers in health programs. Washington, DC: U.S. Department of Health and Human Services; 2006. Available at https://www.cms.gov/reports/downloads/RTC-Leavitt2.pdf

37. Rosenbaum, S.; Shin, P. Migrant and seasonal farmworkers: health insurance coverage and access to care. Washington, DC: Kaiser Commission on Medicaid and the Uninsured; 2005. Available at http://www.kff.org/uninsured/upload/Migrant-and-Seasonal-Farmworkers-Health-InsuranceCoverage-and-Access-to-Care-Executive-Summary.pdf

38. Marinho VC, Higgins JP, Logan S, et al. Topical fluoride (toothpastes, mouthrinses, gels or varnishes) for preventing dental caries in children and adolescents (review). Cochrane Database Syst Rev. 2003; 4:Art. No.: CD002782.10.1002/14651858.CD002782 


\section{Table 1}

\section{Characteristics of Teachers in EHS and MSHS Programs}

\begin{tabular}{|c|c|c|c|}
\hline Variable & Full sample $(n=401) \%$ & EHS $(n=329) \%$ & MSHS $(n=72) \%$ \\
\hline \multicolumn{4}{|l|}{ Dependent variables } \\
\hline Count of parent-focused oral health activities ${ }^{* *}$ & range $=0-4$ & range $=0-4$ & range $=0-4$ \\
\hline (mean, SD) & $(1.44,1.15)$ & $(1.28,1.45)$ & $(2.18,1.60)$ \\
\hline Count of brushing activities $(\mathrm{n}=300)^{* *}$ & range $=0-4$ & range $=0-4$ & range $=1-4$ \\
\hline (mean, SD) & $(2.16,1.11)$ & $(2.02,1.10)$ & $(2.86,0.90)$ \\
\hline \multicolumn{4}{|l|}{ Independent variables } \\
\hline MSHS program & 18.0 & 0 & 100 \\
\hline Received dental health training from $\mathrm{HS}^{* *}$ & 40.4 & 37.1 & 55.3 \\
\hline \multicolumn{4}{|l|}{ Knowledge about... } \\
\hline Amount of toothpaste to use ${ }^{* *}$ & 68.8 & 73.3 & 48.6 \\
\hline Low-income children's increased risk of tooth decay & 78.1 & 79.6 & 70.8 \\
\hline \multicolumn{4}{|l|}{ Value placed on oral health } \\
\hline Low $(\unlhd 0)$ & 26.9 & 25.8 & 31.9 \\
\hline Moderate (11-13) & 47.4 & 46.2 & 52.8 \\
\hline High $(\geq 14)$ & 25.7 & 28.0 & 15.3 \\
\hline \multicolumn{4}{|l|}{ Perceived oral health self-efficacy } \\
\hline Low ( 25 ) & 27.4 & 29.5 & 18.1 \\
\hline Moderate (26-34) & 46.1 & 43.5 & 58.3 \\
\hline High ( $\geq 35)$ & 26.4 & 22.0 & 23.6 \\
\hline Barriers to oral health activities & range $=0-12$ & range $=0-12$ & range $=0-12$ \\
\hline (mean, SD) & $(4.13,3.01)$ & $(4.16,2.95)$ & $(4.04,3.28)$ \\
\hline \multicolumn{4}{|l|}{ Years employed by HS } \\
\hline$<1$ year & 26.0 & 24.3 & 33.3 \\
\hline $1-2$ years & 30.9 & 35.0 & 12.5 \\
\hline 3 or more years & 43.1 & 40.7 & 54.2 \\
\hline \multicolumn{4}{|l|}{ Education level $^{* *}$} \\
\hline High school graduate or less & 14.7 & 12.5 & 25.0 \\
\hline Some college & 59.9 & 58.4 & 66.7 \\
\hline College degree or higher & 25.4 & 29.1 & 8.3 \\
\hline \multicolumn{4}{|l|}{ Race/Ethnicity ${ }^{* *}$} \\
\hline White & 42.4 & 47.7 & 18.0 \\
\hline Black & 37.4 & 37.1 & 38.9 \\
\hline Hispanic/Latina, American Indian, Asian/Pacific Islander, or Other & 20.2 & 15.2 & 43.1 \\
\hline
\end{tabular}

EHS, Early Head Start, MSHS, Migrant \& Seasonal Head Start, HS, Head Start, SD, standard deviation. Differences between staff in MSHS and EHS were obtained using t-tests for continuous independent variables and chi-square tests for categorical independent variables.

* Statistically significant at the $5 \%$ level.

** Statistically significant at the $1 \%$ level.

J Health Care Poor Underserved. Author manuscript; available in PMC 2014 November 20. 


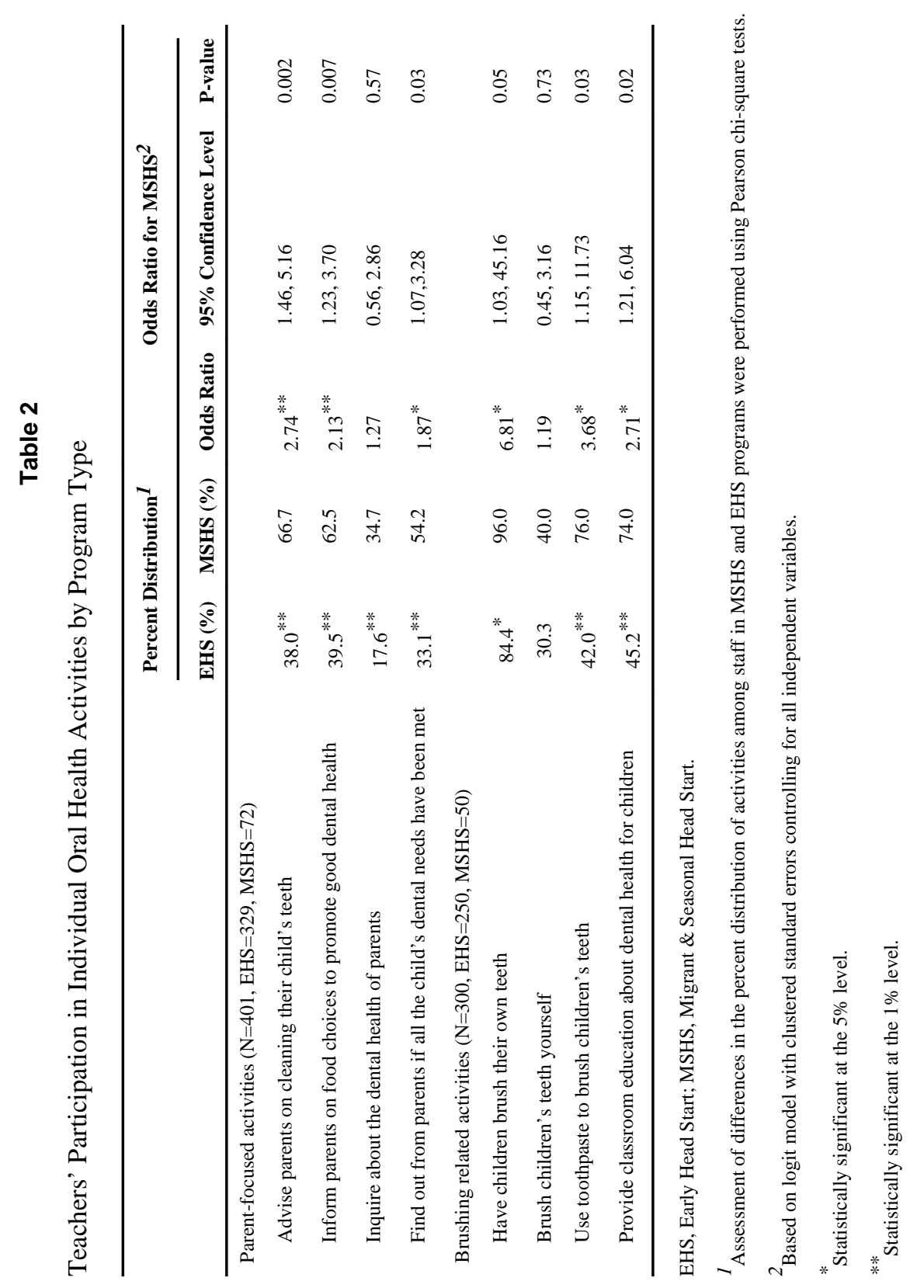

J Health Care Poor Underserved. Author manuscript; available in PMC 2014 November 20. 
